KUL-TF-95/33

hep-th/9510195

October 1995

\title{
Background charges and consistent continuous deformations of $2 d$ gravity theories
}

\author{
Friedemann Brandt ${ }^{1}$, Walter Troost ${ }^{2,3}$ \\ and Antoine Van Proeyen ${ }^{2,4}$ \\ Instituut voor Theoretische Fysica \\ Katholieke Universiteit Leuven \\ Celestijnenlaan 200D \\ B-3001 Leuven, Belgium
}

\begin{abstract}
We discuss all background charges and continuous consistent deformations of standard $2 d$ gravity theories with scalar matter fields. The background charges and those deformations which change nontrivially the gauge symmetries are closely linked, and exist only if the target space has a covariantly constant Killing vector (a null vector for the deformations). The deformed actions provide interesting novel $2 d$ gravity models. Some of them lead to non-critical string theories.
\end{abstract}

${ }^{1}$ Junior fellow of the research council (DOC) of the K.U. Leuven;

E-mail : Friedemann.Brandt@fys.kuleuven.ac.be

${ }^{2}$ Onderzoeksleider, NFWO, Belgium

${ }^{3}$ E-mail : Walter.Troost@fys.kuleuven.ac.be

${ }^{4}$ E-mail : Antoine.VanProeyen@fys.kuleuven.ac.be 
Weyl invariant $2 d$ gravity models have attracted a lot of attention because they underly various systems of physical interest (string theory, twodimensional $\sigma$ - and WZWN-models, conformal field theories). As these models are gauge theories, many of their properties can be analysed by BRST techniques. For a class of such models we computed in [1] (cf. also [2]) the cohomology groups $H^{g}(\mathcal{S})$ of the BRST-Slavnov operator $\mathcal{S}$ in the space of local functionals for all ghost numbers $g$. This letter is devoted to the discussion of the results for $H^{0}(\mathcal{S})$ : we will use them to construct all so-called background charges for these models in the form described in [3] and all continuous deformations of the classical actions which are consistent in the sense that the deformed actions are invariant under possibly deformed gauge transformations [4].

It turns out that both the background charges and those deformations which change nontrivially the form of the gauge transformations exist only for target spaces with a covariantly constant (Killing) vector which must be a null vector in the case of the deformations. Such models deserve special attention also for other reasons: after gauge fixing (e.g. in the conformal gauge) they have a residual (chiral) Kač-Moody symmetry and can thus provide interesting conformal field theories; they allow to gauge target space isometries [5]; and some so-called 'chiral null models' provide solutions of the string equations of motion which are exact to all orders in $\alpha^{\prime}$ [6].

Let us first recall the results of [1] on $H^{0}(\mathcal{S})$. The models in question are fixed solely by the field content and by the gauge symmetries of the classical theory: the fields of the classical theory are the $2 d$ metric $g_{\alpha \beta}=g_{\beta \alpha}$ $(\alpha, \beta \in\{+,-\})$ and an arbitrary number of Weyl invariant scalar 'matter fields' $X^{\mu}(\mu=1, \ldots, D)$; the gauge symmetries are the standard $2 d$ diffeomorphisms and Weyl transformations. The corresponding nilpotent BRST transformations read

$$
\begin{aligned}
s g_{\alpha \beta} & =\xi^{\gamma} \partial_{\gamma} g_{\alpha \beta}+g_{\gamma \beta} \partial_{\alpha} \xi^{\gamma}+g_{\alpha \gamma} \partial_{\beta} \xi^{\gamma}+c g_{\alpha \beta} \\
s \Phi & =\xi^{\alpha} \partial_{\alpha} \Phi \text { for } \Phi \in\left\{X^{\mu}, \xi^{\beta}, c\right\}
\end{aligned}
$$

where the $\xi^{\alpha}$ are the diffeomorphism ghosts and $c$ is the Weyl ghost. As we have shown in [1], any local classical action constructible out of $g_{\alpha \beta}$ and $X^{\mu}$ and invariant under these gauge transformations is, up to boundary terms, of the simple well-known form

$$
S_{c l}=\int \frac{1}{2}\left[\sqrt{g} g^{\alpha \beta} G_{\mu \nu}(X)+\varepsilon^{\alpha \beta} B_{\mu \nu}(X)\right] \partial_{\alpha} X^{\mu} \cdot \partial_{\beta} X^{\nu}
$$


where $g=-\operatorname{det}\left(g_{\alpha \beta}\right)$ (we assume $g>0$ ), $\varepsilon^{+-}=-\varepsilon^{-+}=1$ and $\int \equiv \int d^{2} x$. Hence, different models are distinguished only through the functions $G_{\mu \nu}(X)$ and $B_{\mu \nu}(X)$ occurring in (2) which are symmetric resp. antisymmetric in $\mu, \nu\left(G_{\mu \nu}\right.$ is not assumed to be necessarily invertible). As the gauge algebra is closed and irreducible, the (minimal) solution $S$ of the master equation [7] corresponding to (2) reads

$$
S=S_{c l}-\int\left(s \Phi^{A}\right) \Phi_{A}^{*} ; \quad\left\{\Phi^{A}\right\}=\left\{g_{\alpha \beta}, X^{\mu}, \xi^{\alpha}, c\right\}
$$

where $\Phi_{A}^{*}$ is the antifield of $\Phi^{A}$. The BRST-Slavnov operator $\mathcal{S}$ whose cohomology was computed in [1] is defined on any function(al) $F$ of the fields and antifields through the antibracket [7] of $S$ and $F, \mathcal{S F}:=(S, F)$. $\mathcal{S}$ is a nilpotent antiderivation because $S$ solves the master equation, $(S, S)=0$.

The results of [1] state now that, for any given $G_{\mu \nu}$ and $B_{\mu \nu}$, there are at most two types of nontrivial cohomology classes in $H^{0}(\mathcal{S})$. Those of the first type are obvious ones represented by functionals $W_{(0)}^{0}$ of the same form as the action (2) itself,

$$
W_{(0)}^{0}=\int \frac{1}{2}\left[\sqrt{g} g^{\alpha \beta} N_{\mu \nu}(X)+\varepsilon^{\alpha \beta} F_{\mu \nu}(X)\right] \partial_{\alpha} X^{\mu} \cdot \partial_{\beta} X^{\nu} .
$$

$W_{(0)}^{0}$ is an $\mathcal{S}$-cocycle for any choice of $N_{\mu \nu}$ and $F_{\mu \nu}$ and cohomologically nontrivial unless

$$
N_{\mu \nu}=\mathcal{L}_{H} G_{\mu \nu} \wedge F_{\mu \nu}=\mathcal{L}_{H} B_{\mu \nu}+2 \partial_{[\mu} B_{\nu]}
$$

for some $H^{\mu}(X)$ and $B_{\mu}(X)\left(\mathcal{L}_{H}\right.$ denotes the Lie derivative along $H^{\mu}$ in the target space).

The representatives of $H^{0}(\mathcal{S})$ of the second type depend nontrivially on the antifields. They exist only if $G_{\mu \nu}$ and $B_{\mu \nu}$ allow 'covariantly constant target space vectors' $\zeta_{+}^{\mu}(X)$ or $\zeta_{-}^{\mu}(X)$, solving

$$
\partial_{\mu} \zeta_{\nu \pm}-\left(\Gamma_{\mu \nu, \rho} \pm \frac{1}{2} H_{\mu \nu \rho}\right) \zeta_{ \pm}^{\rho}=0
$$

where $\zeta_{\mu \pm}=G_{\mu \rho} \zeta_{ \pm}^{\rho}$ and

$$
\begin{aligned}
& \Gamma_{\mu \nu, \rho}=\frac{1}{2}\left(\partial_{\mu} G_{\nu \rho}+\partial_{\nu} G_{\mu \rho}-\partial_{\rho} G_{\mu \nu}\right) \\
& H_{\mu \nu \rho}=\partial_{\mu} B_{\nu \rho}+\partial_{\nu} B_{\rho \mu}+\partial_{\rho} B_{\mu \nu} .
\end{aligned}
$$

Note that the $\zeta_{+}$and $\zeta_{-}$are covariantly constant w.r.t. to connections with torsion $\pm(1 / 2) H_{\mu \nu \rho}$ respectively, and that they are special Killing vectors 
of the target space characterized through $G_{\mu \nu}$ and $B_{\mu \nu}$. Introducing bases $\left\{\zeta_{a^{ \pm}}^{\mu}\right\}$ for them $\left(a^{ \pm}=1, \ldots, N^{ \pm}\right)$, the general solutions of (6) can be written as linear combinations of the form $\zeta_{ \pm}^{\mu}(X)=v^{a^{ \pm}} \zeta_{a^{ \pm}}^{\mu}(X)$, where the $v$ 's are constant coefficients. A corresponding basis for the representatives of $H^{0}(\mathcal{S})$ of the second type is provided by the following nontrivial $\mathcal{S}$-cocycles:

$$
W_{a^{ \pm}}^{0}=\int\left(X_{\mu}^{*} \zeta_{a^{ \pm}}^{\mu} C_{ \pm}^{ \pm}-j_{a^{ \pm}}^{\mp} \partial_{ \pm} h_{\mp \mp}\right)
$$

where

$$
\begin{aligned}
h_{ \pm \pm} & =g_{ \pm \pm}\left(g_{+-}+\sqrt{g}\right)^{-1} ; \\
C_{ \pm}^{ \pm} & =\left(\partial_{ \pm} \xi^{ \pm}+h_{\mp \mp} \partial_{ \pm} \xi^{\mp}\right) \\
j_{a^{ \pm}}^{\alpha} & =\left(\sqrt{g} g^{\alpha \beta} \mp \varepsilon^{\alpha \beta}\right) \zeta_{\mu a^{ \pm}} \partial_{\beta} X^{\mu}
\end{aligned}
$$

with $\zeta_{\mu a^{ \pm}}=G_{\mu \nu} \zeta_{a^{ \pm}}^{\nu}$. We note that $h_{ \pm \pm}$are 'Beltrami variables', that the ghost combinations $C_{ \pm}^{ \pm}$satisfy $s C_{ \pm}^{ \pm}=\xi^{\alpha} \partial_{\alpha} C_{ \pm}^{ \pm}$, and that the $j_{a^{ \pm}}^{\alpha}$ are the Noether currents corresponding to the $\zeta_{a^{ \pm}}^{\mu}$ (each $\zeta_{a^{ \pm}}^{\mu}$ generates a rigid symmetry of $S_{c l}$, cf. [四). These currents become chiral in the conformal gauge $\left(g_{+-}=\sqrt{g}, g_{ \pm \pm}=0\right)$. Eq. (8) is reminiscent of similar BRST cocycles in Yang-Mills and Einstein-Yang-Mills theories with $U(1)$-factors found in [8, 9. The role of the rigid symmetries in the BRST cohomology was uncovered in 10].

We consider now a general linear combination of the $\mathcal{S}$-cocycles (8) with arbitrary constant coefficients,

$$
M_{v}^{0}=v^{a^{+}} W_{a^{+}}^{0}+v^{a^{-}} W_{a^{-}}^{0} .
$$

Since $M_{v}^{0}$ is an $\mathcal{S}$-cocycle with ghost number 0 , the antibracket $\left(M_{v}^{0}, M_{v}^{0}\right)$ is automatically an $\mathcal{S}$-cocycle with ghost number one, thanks to the Jacobi identity for the antibracket. Hence, $\left(M_{v}^{0}, M_{v}^{0}\right)$ must be a linear combination of nontrivial representatives of $H^{1}(\mathcal{S})$ modulo an $\mathcal{S}$-coboundary. Using properties of the $\zeta$ 's derived in appendix D of [1] , one finds indeed

$$
\begin{aligned}
\left(M_{v}^{0}, M_{v}^{0}\right) & =2 P_{++}(v) W_{+}^{1}-2 P_{--}(v) W_{-}^{1} \\
- & 2\left(S, W_{v++}^{0}+W_{v--}^{0}+W_{v+-}^{0}\right)
\end{aligned}
$$

where $W_{+}^{1}$ and $W_{-}^{1}$ are nontrivial and cohomologically inequivalent representatives of $H^{1}(\mathcal{S})$ representing the potential matter field independent anomalies [1],

$$
W_{ \pm}^{1}=\mp 2 \int\left(\xi^{ \pm}+h_{\mp \mp} \xi^{\mp}\right)\left(\partial_{ \pm}\right)^{3} h_{\mp \mp}
$$


and $P_{ \pm \pm}(v)$ are constant quadratic forms in the $v$ 's:

$$
P_{ \pm \pm}(v)=\zeta_{ \pm}^{\mu} \zeta_{\mu \pm}=\text { constant } ; \quad \zeta_{ \pm}^{\mu}=v^{a^{ \pm}} \zeta_{a^{ \pm}}^{\mu}
$$

(The constancy of these inner products follows from (6)). The $W^{0}$ 's occurring in (13) are the functionals

$$
\begin{aligned}
W_{v \pm \pm}^{0} & =-P_{ \pm \pm}(v) \int \frac{h_{ \pm \pm}\left(\partial_{ \pm} h_{\mp \mp}\right)^{2}}{1-h_{++} h_{--}} \\
W_{v+-}^{0} & =\int \frac{2 P_{+-}(v, X) \partial_{+} h_{--} \cdot \partial_{-} h_{++}}{1-h_{++} h_{--}}
\end{aligned}
$$

where $P_{+-}(v, X)$ is the inner product of $\zeta_{+}^{\mu}$ and $\zeta_{-}^{\mu}$ which is in general not constant, contrary to (15), but still annihilated by the operators $\zeta_{ \pm}^{\mu} \partial_{\mu}$ (see Eq. (D.21) of [1]),

$$
P_{+-}(v, X)=\zeta_{+}^{\mu} \zeta_{\mu-} ; \quad \zeta_{ \pm}^{\mu} \partial_{\mu} P_{+-}(v, X)=0 .
$$

We will now discuss (13) and show that it leads to background charges and continuous deformations. It is straightforward to verify that

$$
S_{\tau}(v)=S+\tau M_{v}^{0}+\tau^{2}\left(W_{v++}^{0}+W_{v--}^{0}+W_{v+-}^{0}\right)
$$

satisfies for any value of $\tau$

$$
\left(S_{\tau}(v), S_{\tau}(v)\right)=2 \tau^{2}\left(P_{++}(v) W_{+}^{1}-P_{--}(v) W_{-}^{1}\right),
$$

which holds at $\tau=0$ by construction of $S$, linear in $\tau$ by definition of $M_{v}^{0}$, quadratic in $\tau$ due to (13), at $\tau^{3}$ by (18) and at $\tau^{4}$ because the functionals (16) and (17) do not depend on antifields. We use this result in two different ways:

a) Suppose that $\hbar\left(a_{+} W_{+}^{1}+a_{-} W_{-}^{1}\right)$ occurs as a one-loop anomaly of the theory. Suppose further that there are coefficients $\bar{v}$ satisfying $2 P_{ \pm \pm}(\bar{v})=$ $\mp a_{ \pm}$. According to (13) we can then formally cancel this anomaly (up to terms of higher order in $\hbar$ ) by using as action $S_{\sqrt{\hbar}}(\bar{v})$. Thus $M_{\bar{v}}^{0}$ represents a background charge, cf. [3]. In particular one may cancel in this way the famous Weyl anomaly whose coefficient is proportional to $(D-26)$. Namely up to the coefficient this anomaly is represented by $\int c \sqrt{g} R$ which is cohomologically equivalent to $W_{+}^{1}-W_{-}^{1}$. 
b) The second application aims to construct a continuous deformation of the classical action. This is applicable for target spaces with a non-positive definite metric $G_{\mu \nu}(X)$. Suppose that a set of $\hat{v}$ 's solves nontrivially

$$
P_{++}(\hat{v})=P_{--}(\hat{v})=0 .
$$

This requires the existence of a covariantly constant null vector $\hat{\zeta}_{+}^{\mu} \equiv \hat{v}^{a^{+}} \zeta_{a^{+}}^{\mu}$ or $\hat{\zeta}_{-}^{\mu} \equiv \hat{v}^{a^{-}} \zeta_{a^{-}}^{\mu}$. Then (20) says that $S_{\tau}(\hat{v})$ satisfies the master equation for any value of $\tau$ and is thus a consistent continuous deformation of $S$ with deformation parameter $\tau$. In fact it is a nontrivial one, i.e. it cannot be obtained from (3) through mere local field redefinitions because $M_{\hat{v}}^{0}$ is a nontrivial $\mathcal{S}$-cocycle. Henceforth $S_{\tau}$ will always denote $S_{\tau}(\hat{v})$. Let us now briefly discuss it. It is of the form

$$
S_{\tau}=S_{c l, \tau}-\int\left(s_{\tau} \Phi^{A}\right) \Phi_{A}^{*}
$$

where $S_{c l, \tau}$ is the antifield independent part of $S_{\tau}$. This part is the deformation of the classical action (2). Since (21) implies $W_{\hat{v} \pm \pm}^{0}=0, S_{c l, \tau}$ is explicitly given by

$$
\begin{aligned}
S_{c l, \tau} & =S_{c l}+2 \int \frac{\tau\left(\mathcal{L}^{+}+\mathcal{L}^{-}\right)+\tau^{2} \mathcal{L}^{+-}}{1-h_{++} h_{--}} ; \\
\mathcal{L}^{ \pm} & =-\partial_{ \pm} h_{\mp \mp} \cdot \hat{\zeta}_{\mu \pm}(X)\left(\partial_{ \pm}-h_{ \pm \pm} \partial_{\mp}\right) X^{\mu} ; \\
\mathcal{L}^{+-} & =\hat{\zeta}_{+}^{\mu}(X) \hat{\zeta}_{\mu-}(X) \partial_{+} h_{--} \cdot \partial_{-} h_{++}
\end{aligned}
$$

where we rewrote (111) in terms of the Beltrami variables. Note that $S_{c l, \tau}$ is in general not 'left-right symmetric', contrary to $S_{c l}$ (left-right symmetry is obtained only for the choice $\hat{\zeta}_{+}^{\mu}=\hat{\zeta}_{-}^{\mu}$ ). (20) implies that $S_{c l, \tau}$ is invariant under deformed BRST transformations (and corresponding deformed gauge transformations) given by

$$
\begin{aligned}
s_{\tau} X^{\mu} & =\xi^{\alpha} \partial_{\alpha} X^{\mu}+\tau\left(C_{+}^{+} \hat{\zeta}_{+}^{\mu}+C_{-}^{-} \hat{\zeta}_{-}^{\mu}\right) ; \\
s_{\tau} \Phi & =s \Phi \text { for } \Phi \in\left\{g_{\alpha \beta}, \xi^{\alpha}, c\right\} .
\end{aligned}
$$

The fact that (22) is linear in the antifields indicates that the deformed gauge transformations still have a closed algebra. In fact this algebra agrees with the original one, as the BRST transformations of the ghosts remain undeformed, cf. (25). Hence, even though the gauge transformations themselves 
get nontrivially deformed, their commutation relations do not change (one may check this using $\hat{\zeta}_{+}^{\nu} \partial_{\nu} \hat{\zeta}_{-}^{\mu}-\hat{\zeta}_{-}^{\nu} \partial_{\nu} \hat{\zeta}_{+}^{\mu}=0$, cf. appendix D of [1]).

In order to make contact with results known in the literature and to give some insight into the physical content of the deformed models, we will now specialise to the case

$$
\operatorname{det}\left(G_{\mu \nu}\right) \neq 0, \quad B_{\mu \nu}=0 .
$$

In this case we can obviously choose $\zeta_{a^{+}}^{\mu}=\zeta_{a^{-}}^{\mu}$. Nevertheless the deformed actions (23) will not be left-right symmetric unless we choose also $\hat{v}^{a^{+}}=\hat{v}^{a^{-}}$ which yields

$$
\hat{\zeta}_{+}^{\mu}=\hat{\zeta}_{-}^{\mu} \equiv \hat{\zeta}^{\mu}
$$

Let us now analyse the deformed theory in some more detail for this special choice. Recall that we must impose $\hat{\zeta}^{\mu} \hat{\zeta}_{\mu}=0$, cf. (21). This implies due to (27) that the part $\mathcal{L}^{+-}$of $S_{c l, \tau}$ vanishes. (6) implies in this case

$$
\begin{gathered}
\partial_{[\mu} \hat{\zeta}_{\nu]}=0 \Leftrightarrow \hat{\zeta}_{\mu}(X)=\partial_{\mu} \Lambda(X) \quad(D \neq 1) \\
\wedge \quad \partial_{\mu} \partial_{\nu} \Lambda-\Gamma_{\mu \nu}{ }^{\rho} \partial_{\rho} \Lambda=0 .
\end{gathered}
$$

Using partial integrations (and Eq. (A.12) of [1]) one straightforwardly verifies that (23) reduces in the special case (26) and for the choice (27) to

$$
\begin{aligned}
S_{c l, \tau}= & \int \sqrt{g}\left[\frac{1}{2} g^{\alpha \beta} G_{\mu \nu}(X) \partial_{\alpha} X^{\mu} \cdot \partial_{\beta} X^{\nu}\right. \\
& \left.-\tau \Lambda(X) R-\tau g^{\alpha \beta} \partial_{\alpha} L \cdot \partial_{\beta} \Lambda(X)\right]
\end{aligned}
$$

where $R$ is the $2 d$ Riemann curvature scalar constructed out of $g_{\alpha \beta}$, and $L$ is the useful quantity

$$
L=\ln \frac{\sqrt{g}}{1-h_{++} h_{--}}=\ln \frac{\sqrt{g}+g_{+-}}{2} .
$$

One may now verify that the local field redefinition

$$
Y^{\mu}(X, L, \tau)=\exp \left[-\tau L \hat{\zeta}^{\nu}(X) \partial / \partial X^{\nu}\right] X^{\mu}
$$

casts (30) in a simpler form which is regular even for $g_{+-}+\sqrt{g}=0$,

$$
S_{c l, \tau}=\int \sqrt{g}\left[\frac{1}{2} g^{\alpha \beta} G_{\mu \nu}(Y) \partial_{\alpha} Y^{\mu} \cdot \partial_{\beta} Y^{\nu}-\tau \Lambda(Y) R\right]
$$


where $G_{\mu \nu}$ and $\Lambda$ are the same functions as in (30) (but note that their arguments are now the $Y^{\prime}$ 's instead of the $X$ 's). To obtain this result, one uses

$$
\begin{aligned}
& \exp \left[-\tau L \hat{\zeta}^{\nu}(X) \partial / \partial X^{\nu}\right] f(X)=f(Y) \\
& \Rightarrow \quad \hat{\zeta}^{\nu}(X)\left(\partial Y^{\mu} / \partial X^{\nu}\right)=\hat{\zeta}^{\mu}(Y),
\end{aligned}
$$

for any function $f(X)$, and further that

$$
G_{\mu \nu}(X) \frac{\partial X^{\mu}}{\partial Y^{\rho}} \frac{\partial X^{\nu}}{\partial Y^{\sigma}}=G_{\rho \sigma}(Y) ; \quad \Lambda(X)=\Lambda(Y)
$$

where the first identity holds because $\hat{\zeta}$ is a Killing vector and the second one because it is a null vector. The deformed BRST transformation of $Y^{\mu}$ reads

$$
s_{\tau} Y^{\mu}=\xi^{\alpha} \partial_{\alpha} Y^{\mu}-\tau c \hat{\zeta}^{\mu}(Y)
$$

which follows also immediately from (32) due to (34) and

$$
s_{\tau} L=s L=\xi^{\alpha} \partial_{\alpha} L+c+C_{+}^{+}+C_{-}^{-} .
$$

Finally we show that in a model described at classical level by a deformed action (33) one may cancel the matter field independent Weyl anomaly by a counterterm. To that end we choose a function $\varphi(X)$ such that

$$
\hat{\zeta}^{\mu}(X) \partial_{\mu} \varphi(X)=1 \text {. }
$$

Such a function $\varphi$ always exists. This is easily seen in a target space parametrization chosen such that $\hat{\zeta}^{\mu}$ has the components $(1,0, \ldots, 0)$ (i.e. the integral curves of $\hat{\zeta}^{\mu}$ are chosen as first coordinate lines in that parametrization). Then (38) requires $\varphi(X)=X^{1}+f\left(X^{2}, \ldots, X^{D}\right)$ where the function $f$ can be chosen arbitrarily (i.e. $\varphi$ is not unique).

Using (32), (36) and (38) one now easily verifies

$$
\begin{aligned}
\varphi(Y) & =\varphi(X)-\tau L ; \\
s_{\tau} \varphi(Y) & =\xi^{\alpha} \partial_{\alpha} \varphi(Y)-\tau c .
\end{aligned}
$$

(40) implies that $\varphi(Y)$ plays in the deformed theory the role of a 'Liouville field' as one has

$$
\begin{aligned}
& s_{\tau} W_{\varphi}^{0}=\tau^{2} \int c \sqrt{g} R \\
& W_{\varphi}^{0}=\int \sqrt{g}\left[\frac{1}{2} g^{\alpha \beta} \partial_{\alpha} \varphi(Y) \cdot \partial_{\beta} \varphi(Y)-\tau \varphi(Y) R\right] .
\end{aligned}
$$


This shows that for $\tau \neq 0$ the matter field independent Weyl anomaly $\int c \sqrt{g} R$ may be cancelled, say at the one-loop level, by a counterterm proportional to $\hbar W_{\varphi}^{0}$. That is impossible in the undeformed theory (i.e. for $\tau=0$ ) because $\int c \sqrt{g} R$ is nontrivial in $H^{1}(\mathcal{S})$ and thus cannot be cancelled through the $\mathcal{S}$ variation of any local functional [1] (this is reflected by the occurrence of $\tau$ on the r.h.s. of (41)).

A simple example to which the above construction applies is of course a flat target space with $G_{\mu \nu}=\operatorname{diag}(-1,1, \ldots, 1)$. In this case the covariantly constant vectors are just constants and thus $\Lambda(X)$ is linear in the $X^{\mu}$. E.g. one can choose $\Lambda(X)=X^{1}+X^{2}$ and $\varphi(X)=-X^{1}$ which gives $Y^{1}=X^{1}+\tau L$, $Y^{2}=X^{2}-\tau L$ and $Y^{\mu}=X^{\mu}$ for $\mu>2$. Examples for curved target spaces with covariantly constant null vectors (and nonvanishing $B_{\mu \nu}$ ) can be found in [6].

We conclude this letter with the following remarks:

1. We stress again that background charges and consistent continuous deformations changing nontrivially the gauge transformations exist only for target spaces possessing covariantly constant (null) vectors solving (6). Namely otherwise there are no representatives of $H^{0}(\mathcal{S})$ depending nontrivially on antifields [1].

2. Eq. (12) does not give the most general form of the background charges because one can still add a functional (II) to $M_{v}^{0}$. This results in additional contributions on the r.h.s. of Eqs. (13) and (20) because in general the antibracket $\left(M_{v}^{0}, W_{(0)}^{0}\right)$ contains matter field dependent candidate anomalies of the form given in Eq. (10.8) in [1]. Hence, even such anomalies can possibly be cancelled through background charges, but, again, only in presence of solutions of (6).

3. Similarly, functionals (4) may contribute to continuous deformations of (2) if there are continuous families $G_{\mu \nu}(X, \tau)=G_{\mu \nu}(X)+\tau N_{\mu \nu}(X)+\ldots$ and $B_{\mu \nu}(X, \tau)=B_{\mu \nu}(X)+\tau F_{\mu \nu}(X)+\ldots$ with at least one corresponding continuous set of covariantly constant null vectors.

4. The $2 d$ gravity models obtained from deformed classical actions (23) are new. This is evident for left-right asymmetric actions (23) but it holds also for the left-right symmetric ones. Concerning the latter we note that for instance (33) must not be confused with the celebrated 'dilaton action' even though it has the same form as the latter. The crucial difference is that (33) is gauge invariant at classical level, contrary to the dilaton action (recall that $\Lambda$ has to satisfy Eq. (29) and is thus not the 'dilaton' as the latter is determined by the cancellation condition for matter field dependent Weyl 
anomalies, cf. [11] and section 11 of [四).

5. In a deformed theory $\tau$ plays the role of a coupling constant. For $\tau \neq 0$ one might obtain non-critical string theories because the matter field independent Weyl anomaly might be cancelled without introducing a new ('Liouville') field by hand (cf. above discussion of (41)). This points out once more that the deformed theory is different from the original one and has physically interesting properties.

6. An interesting interpretation of a deformed action (23) would arise if it would represent a quantum deformation of a corresponding classical action (2), i.e. if $\tau \sim \hbar$. In that case the $\tau$-dependent term in Eq. (24) would be a quantum correction of order $\hbar$ to the classical BRST-transformation. As the deformed gauge transformations have the same algebra as the original ones, the theory would satisfy the structural constraints of [12].

\section{Acknowledgments.}

We thank O. Lechtenfeld for pointing out to us ref. [6]. This work was carried out in the framework of the European Community Research Programme "Gauge theories, applied supersymmetry and quantum gravity", with a financial contribution under contract SC1-CT92-0789.

\section{References}

[1] F. Brandt, W. Troost and A. Van Proeyen, preprint KUL-TF-95/17, hep-th/9509035.

[2] F. Brandt, W. Troost and A. Van Proeyen, in Geometry of Constrained Dynamical Systems (Cambridge Univ. Press, Cambridge, 1995);

W. Troost and A. Van Proeyen, in Strings and Symmetries, Lecture Notes in Physics, Vol. 447 (Springer-Verlag, New York, 1995).

[3] F. De Jonghe, R. Siebelink and W. Troost, Phys. Lett. B306, 295 (1993);

S. Vandoren and A. Van Proeyen, Nucl. Phys. B411, 257 (1994).

[4] G. Barnich and M. Henneaux, Phys. Lett. B311, 123 (1993).

[5] C. M. Hull and B. Spence, Phys. Lett. B232, 204 (1989). 
[6] G. T. Horowitz, A. A. Tseytlin, Phys. Rev. D51, 2896 (1995);

A. A. Tseytlin, Class. Quant. Grav. 12, 2365 (1995). hep-th/9505052.

[7] I. A. Batalin and G. A. Vilkovisky, Phys. Lett. B102, 27 (1981); Phys. Rev. D28, 2567 (1983) (E:D30, 508 (1984)).

[8] G. Barnich and M. Henneaux, Phys. Rev. Lett. 72, 1588 (1994);

G. Barnich, F. Brandt and M. Henneaux, Commun. Math. Phys. 174,93 (1995). hep-th/9405194

[9] G. Barnich, F. Brandt and M. Henneaux, Nucl. Phys. B455 (1995) 357.

[10] G. Barnich, F. Brandt and M. Henneaux, Commun. Math. Phys. 174, 57 (1995). hep-th/9405109

[11] E. Fradkin and A. Tseytlin, Nucl. Phys. B261 (1985) 1;

C. G. Callan, D. Friedan, E. J. Martinec and M. J. Perry, Nucl. Phys. B262 (1985) 593.

[12] J. Gomis and S. Weinberg, preprint RIMS-1036, UTTG-18-95, hepth/9510087. 